\title{
Mechanosensory Activation of a Motor Circuit by Coactivation of Two Projection Neurons
}

\author{
Mark P. Beenhakker and Michael P. Nusbaum \\ Department of Neuroscience, University of Pennsylvania School of Medicine, Philadelphia, Pennsylvania 19104
}

\begin{abstract}
Individual neuronal circuits can generate multiple activity patterns because of the influence of different projection neurons. However, in most systems it has been difficult to identify and assess the relative contribution of all upstream neurons responsible for the activation of any single activity pattern by a behaviorally relevant stimulus. To elucidate this issue, we used the stomatogastric nervous system (STNS) of the crab. The STNS includes the gastric mill (chewing) motor circuit in the stomatogastric ganglion (STG) and no more than 20 projection neurons that innervate the STG. We previously identified at least some (four) of the projection neurons that are activated directly by the ventral cardiac neuron $(\mathrm{VCN})$ system, a population of mechanosensory neurons that activates the gastric mill circuit. Here we show that two of these projection neurons, the previously identified modulatory commissural neuron 1 (MCN1) and commissural projection neuron 2 (CPN2), are necessary and likely sufficient for the initiation/maintenance of the VCN-elicited gastric mill rhythm. Selective inactivation of either MCN1 or CPN2 still enabled a VCN-elicited gastric mill rhythm. However, because MCN1 and CPN2 have different actions on gastric mill neurons, these manipulations resulted in rhythms distinct from each other and from that occurring in the intact system. After removal of both MCN1 and CPN2, VCN stimulation failed to activate the gastric mill rhythm. Selective conjoint stimulation of $\mathrm{MCN} 1$ and $\mathrm{CPN} 2$, approximating their $\mathrm{VCN}$-elicited activity patterns and firing frequencies, elicited a VCN-like gastric mill rhythm. Thus the VCN mechanosensory system elicits the gastric mill rhythm via its activation of a subset of the relevant projection neurons.
\end{abstract}

Key words: stomatogastric ganglion; neuromodulation; gastric mill circuit; central pattern generator; rhythm; mechanoreceptor

\section{Introduction}

A complete understanding of how sensory information is processed to generate specific behaviors remains elusive in most neuronal systems. In some systems experimental results indicate that individual sensory inputs diverge to influence many upstream neurons so that the resulting network output results from the pooled activity of a population of these generally not well characterized neurons (Sparks, 1988; Georgopoulos, 1995; Kristan and Shaw, 1997; Lewis, 1999; Pouget et al., 2000; Gold and Shadlen, 2001). This hypothesis, often labeled "population coding," has been suggested to underlie those behaviors that involve a directional response to sensory stimuli (Lewis, 1999), as well as behaviors that are categorically distinct (Kristan and Shaw, 1997). Regardless of the behavioral context, the idea that information used by neuronal networks is represented within the activity levels of upstream neurons is likely to be fundamental to our understanding of how any neuronal network produces a particular activity pattern. Despite the appeal of this coding scheme, it has been difficult to assess how sensory information is processed by the descending neurons that transmit this code.

Received May 3, 2004; revised June 17, 2004; accepted June 17, 2004

This work was supported by National Institute of Neurological Disorders and Stroke Grants NS42813 and NS29436 (M.P.N.) and F31-NS41894 (M.P.B.). We thank Dr. Josh Gold for his guidance on statistical measures.

Correspondence should be addressed to Dr. Michael P. Nusbaum, Department of Neuroscience, 215 Stemmler Hall, University of Pennsylvania School of Medicine, Philadelphia, PA 19104-6074. E-mail: nusbaum@mail.med.upenn.edu. DOI:10.1523/JNEUROSCI.1682-04.2004

Copyright $\odot 2004$ Society for Neuroscience $\quad 0270-6474 / 04 / 246741-10 \$ 15.00 / 0$
We are addressing this issue by using the stomatogastric nervous system (STNS) of the crab Cancer borealis. The STNS, which consists of four ganglia plus their connecting and peripheral nerves, contains a set of central pattern-generating (CPG) circuits that control aspects of feeding (Nusbaum and Beenhakker, 2002). One of these ganglia, the stomatogastric ganglion (STG), contains two distinct but interacting rhythmically active circuits responsible for the chewing (gastric mill circuit) and filtering (pyloric circuit) of food (Harris-Warrick et al., 1992). The STG circuits receive input from $\sim 20$ projection neurons in the paired commissural ganglia (CoGs) and oesophageal ganglion (OG) (Coleman et al., 1992). In C. borealis many of these projection neurons are not spontaneously active, including three previously identified CoG projection neurons, modulatory commissural neurons 1, 5, and 7 (MCN1, MCN5, MCN7) (Norris et al., 1996; Nusbaum et al., 2001). A fourth projection neuron, commissural projection neuron 2 (CPN2), does show some spontaneous activity (Norris et al., 1994).

The actions of several sensory systems on the STG circuits have been documented (Simmers and Moulins, 1988a,b; Katz et al., 1989; Hooper et al., 1990; Meyrand et al., 1994; Combes et al., 1999a; Beenhakker et al., 2004). One of these systems, the ventral cardiac neuron ( $\mathrm{VCN}$ ) mechanosensory system, is a population of sensory neurons innervating the wall of the cardiac sac stomach compartment that are hypothesized to detect foregut distention (Beenhakker et al., 2004). When activated for 1-2 min, the VCN system elicits a gastric mill rhythm that persists for tens of 
minutes. The activation of this rhythm results from $\mathrm{VCN}$ actions in the CoGs. The VCNs have synaptic effects on several CoG neurons, including MCN1, MCN5, MCN7, and CPN2 (Beenhakker et al., 2004). Here we demonstrate that, despite these relatively widespread actions, VCN activation of the gastric mill rhythm results mainly and perhaps entirely from its long-lasting activation of MCN1 and CPN2.

Some of this work has appeared in abstract form (Beenhakker et al., 2000).

\section{Materials and Methods}

Animals/experimental preparation. C. borealis (Jonah crabs) were supplied by Commercial Lobster and Seafood Company (Boston, MA) and the Marine Biological Laboratory (Woods Hole, MA). Before experimentation the crabs were maintained in commercial tanks filled with chilled $\left(10^{\circ} \mathrm{C}\right)$, filtered, and recirculating artificial seawater. Immediately before dissection the crabs were anesthetized by packing them into ice for at least $30 \mathrm{~min}$. Then the dorsal carapace was removed, after which the foregut was dissected and removed. The isolated foregut subsequently was maintained in chilled C. borealis saline (see below) while the STNS was dissected from it. Finally, the isolated STNS was pinned down in a silicone elastomer-lined (Sylgard 184, K. R. Anderson, Santa Clara, CA) Petri dish. During each experiment the STNS was superfused continuously $(7-12 \mathrm{ml} / \mathrm{min})$ with chilled $\left(10-12^{\circ} \mathrm{C}\right)$ saline.

Solutions. C. borealis saline included (in mM): $440 \mathrm{NaCl}, 26 \mathrm{MgCl}_{2}, 11$ $\mathrm{KCl}, 13 \mathrm{CaCl}_{2}, 10$ Trizma base, and 5 maleic acid, $\mathrm{pH}$ 7.4-7.6.

Electrophysiology. All experiments were performed on the isolated STNS (see Fig. 1A). At the onset of each experiment the neurons were identified by using routine methods described previously (Beenhakker et al., 2004). Briefly, neurons were identified by their patterns of activity, interactions with other neurons, and axonal branching patterns (Weimann et al., 1991; Norris et al., 1994; Blitz and Nusbaum, 1997; Blitz et al., 1999). All but one gastric mill circuit neuron [interneuron 1 (Int1] in the STG are also motor neurons that project via the posterior STG nerves to innervate their target muscles. Int1 is an interneuron that projects via the stomatogastric nerve $(s t n)$ and superior oesophageal nerves (sons) to both CoGs.

Neuronal activity levels were monitored via intracellular and extracellular recordings. Intracellular recordings were achieved by desheathing the STNS ganglia and visualizing individual neurons by illuminating the ganglia from below with light transmitted through a dark-field condenser (Nikon, Tokyo, Japan). Intrasomatic recordings were performed with sharp glass microelectrodes $(15-30 \mathrm{M} \Omega$ ) filled with 4 M potassium acetate and $20 \mathrm{~mm} \mathrm{KCl}$. Intra-axonal recordings of neurons also were performed in some experiments by using sharp glass microelectrodes (20-30 M $\Omega$ ) filled with $1 \mathrm{~m} \mathrm{KCl} \mathrm{(Coleman} \mathrm{et} \mathrm{al.,} \mathrm{1995).} \mathrm{All} \mathrm{intracellular}$ signals were recorded with Axoclamp 2B amplifiers (Axon Instruments, Foster City, CA). Intracellular current injections were performed by using single-electrode discontinuous current clamp (DCC) with sample rates between 2 and $3 \mathrm{kHz}$. Each extracellular recording was obtained by using two stainless steel wire electrodes, one of which was pressed into the Sylgard within a petroleum jelly well (Vaseline, Chesebrough-Ponds, Greenwich, CT) that isolated a section of a STNS nerve from the bath. The second, reference, electrode was placed in the common bath. A differential signal between the recording and reference electrode was recorded, filtered, and amplified (stage 1, model 1700 AC amplifier, A-M Systems, Carlsborg, WA; stage 2, model 410 AC/DC amplifier, Brownlee Precision, Santa Clara, CA). Some neurons were stimulated selectively and extracellularly by placing the pair of extracellular recording wires into a stimulus isolation unit (model SIU 5, Astro-Med/Grass Instruments, West Warwick, RI) that was controlled by a stimulator (model S88, Astro-Med/Grass Instruments).

Data acquisition. All data were collected in parallel by two independent systems. One of these systems was a chart recorder (MT-95000, AstroMed). The second system used SPIKE2 data acquisition software provided by Cambridge Electronic Design (Cambridge, UK) to digitize $(\sim 5$ $\mathrm{kHz}$ ) the data for collection onto a personal computer. Data analyses were conducted on the digitized data by using a custom-written SPIKE2 program called "The Crab Analyzer" (freely available at http://www.neurobiologie. $\mathrm{de} /$ ) that determines the activity levels and phase relationships of neurons. This program defines burst duration as the duration (sec) between the onset of the first and last action potential within an impulse burst and defines the firing rate of a neuron as the number of action potentials minus one, divided by the burst duration.

The cycle period of each gastric mill rhythm analyzed herein corresponded to the duration (sec) between the onset of two successive lateral gastric (LG) neuron bursts. During a gastric mill rhythm the relationships among the gastric mill neuron bursts were expressed in terms of phase (i.e., the fraction of a normalized gastric mill cycle during which a neuron begins and ends its burst). Thus phase relationships among gastric mill neurons were determined relative to a gastric mill cycle as defined by the onset of one LG neuron burst (beginning of the cycle) and the onset of the subsequent LG neuron burst (end of the cycle). Each datum in a data set corresponds to the mean of five consecutive impulse bursts. Nonparametric statistical analyses (Wilcoxon signed rank test and Kruskal-Wallis test) were performed with the use of SigmaStat 3.0 (SPSS, Chicago, IL). Data are expressed as the mean \pm SD. Data figures were made by incorporating SPIKE2-generated bitmap figures into the Adobe Photoshop (Adobe, San Jose, CA) and PowerPoint (Microsoft, Seattle, WA) graphics programs. Finally, because every gastric mill neuron cannot be recorded in every experiment, the number of observations for any given result $(n)$ differs among the neuron types.

Experimental manipulations. Previously described VCN stimulation protocols were used to elicit the gastric mill rhythm (Beenhakker et al., 2004). Briefly, the stimulation protocol consisted of a train of 10 6-secduration bursts of stimuli (intraburst frequency, $15 \mathrm{~Hz}$ ) delivered once every $16 \mathrm{sec}$ (interburst frequency, $0.06 \mathrm{~Hz}$ ). In experiments in which the influence of the MCN1 and CPN2 projection neurons was eliminated to determine the consequences for the gastric mill rhythm, one CoG was removed to simplify the experiment. One CoG is sufficient to enable the VCNs to elicit the gastric mill rhythm (Beenhakker et al., 2004). MCN1 projects its axon through the inferior oesophageal nerve (ion) to reach the gastric mill circuit within the STG (see Fig. $1 A$ ), so the influence of this projection neuron on the gastric mill circuit was eliminated readily by transecting this nerve. Although one other projection neuron (MCN5) (Coleman et al., 1992; Norris et al., 1996) also takes this route to the STG and thus is eliminated with ion nerve transection, the activity level of MCN5 after VCN stimulation is too low to have an impact on the gastric mill rhythm (Norris et al., 1996; Beenhakker et al., 2004). Nonetheless, because of the presence of MCN5 in the ion, we were motivated to determine whether MCN1 and CPN2 activity was sufficient to recapitulate the VCN-elicited gastric mill rhythm (see below and Results). The axons of the other $\sim 20$ STG-projecting CoG neurons, including CPN2, project to the STG through the sons (Coleman et al., 1992) (see Fig. 1A). Thus we selectively eliminated CPN2 activity by injecting hyperpolarizing current through an intra-axonal CPN2 recording within the stn, near the entrance to the STG (Blitz and Nusbaum, 1997) (see Fig. 1A). We documented the elimination of CPN2 influence in the STG during a hyperpolarization by monitoring the presence/absence of EPSPs in the gastric mill (GM) neuron. All EPSPs in GM originate from CPN2 (Norris et al., 1994). Finally, in some experiments the activity patterns of MCN1 and CPN2 were imposed on the gastric mill circuit. MCN1 was activated selectively by extracellular ion stimulation because the ion stimulus voltage required to activate $\mathrm{MCN} 1$ is lower than that of MCN5 (Bartos and Nusbaum, 1997). Selective activation of CPN2 was performed by intraaxonal depolarizing current injection into its stn axon. Programs that controlled nerve stimulation and current injections were written by using SPIKE2 output Sequencer code.

\section{Results}

\section{The VCN-elicited gastric mill rhythm is influenced by MCN1 and CPN2}

The VCN mechanosensory system has synaptic actions on all identified projection neurons in the CoGs, including MCN1, MCN5, MCN7, and CPN2, with each target neuron exhibiting a stereotyped response to VCN stimulation (Beenhakker et al., 
2004). At least some of these actions were likely to be pivotal to the VCN activation of the gastric mill rhythm, because removal of the CoGs eliminates gastric mill rhythm activation (Beenhakker et al., 2004). As an initial assessment of the potential contribution of these CoG projection neurons to the VCN activation of the gastric mill rhythm, we eliminated the influence of individual projection neurons via intrasomatic hyperpolarizing current injection during VCN-elicited rhythms. Removing the influence of either MCN5 $(n=3)$ or MCN7 $(n=2)$ had no effect on the ongoing VCN-elicited gastric mill rhythm (data not shown). This result was not too surprising because during these rhythms the activity level of these two projection neurons is weak (MCN5, $\leq 3$ $\mathrm{Hz}$; MCN7, $\leq 1 \mathrm{~Hz}$ ) or absent (Beenhakker et al., 2004). We found that only selective removal of MCN1 or CPN2 altered VCN-elicited gastric mill rhythms (see below).

Suppressing the activity of either MCN1 or CPN2 by intrasomatic injection of hyperpolarizing current changed the gastric mill rhythm that resulted from VCN stimulation (data not shown). After these initial experiments we took a different approach to manipulating the MCN1 and CPN2 influence on the VCN-elicited gastric mill rhythm because some CoG neurons, including MCN1 and CPN2, exhibit electrical coupling to other CoG neurons (M. P. Beenhakker and M. P. Nusbaum, unpublished observations). This coupling leaves open the possibility that hyperpolarizing one neuron also might hyperpolarize other, unknown neurons, making it impossible to determine the specific influence of the manipulated neuron on the VCNinfluenced gastric mill circuit. Therefore, we used two experimental approaches to eliminate selectively the influence of MCN1 and CPN2 on the gastric mill rhythm. First, we selectively eliminated the influence of MCN1 during VCN-elicited gastric mill rhythms by transecting the ion. This transection selectively affects MCN1 because MCN5 is the only other CoG neuron to project to the STG via the ion (see Materials and Methods) (Coleman et al., 1992; Norris et al., 1996). Second, we selectively eliminated the influence of CPN2 by injecting sufficient hyperpolarizing current into its stn axon, thereby suppressing the propagation of its action potentials into the STG and eliminating its postsynaptic actions on its STG target neurons (e.g., GM neuron) (see Fig. 2). The hyperpolarization of the CPN2 axon had no influence on the CPN2 actions in the CoG because the intraaxonal recording site of CPN2 is electrotonically distant from the CoG (Coleman and Nusbaum, 1994).

\section{Selective removal of $\mathrm{CPN} 2$ alters the $\mathrm{VCN}$-elicited gastric mill rhythm}

Removing the influence of the CPN2 projection neuron did not prevent the generation of a VCN-elicited gastric mill rhythm, but the resulting rhythm was different. The changed rhythm was altered in a manner consistent with the removal of known CPN2 actions (Fig. 1 B) (Norris et al., 1994). For example, CPN2 excites the lateral gastric (LG) and gastric mill (GM) neurons, and it inhibits the dorsal gastric (DG) and medial gastric (MG) neurons. Correspondingly, removal of CPN2 influence resulted in a VCN-elicited gastric mill rhythm that did not include GM neuron activity ( $n=7$ of 8 preparations) and was characterized by briefer LG neuron impulse bursts $(p<0.01 ; n=8)$ with fewer action potentials per burst $(p<0.001 ; n=8)$ (Figs. 2,3$)$. The LG neuron burst also occupied a smaller fraction of the gastric mill cycle (e.g., reduced duty cycle, $p<0.01 ; n=8$ ). Removal of the CPN2 inhibitory actions on the MG and DG neurons was also evident. Relative to control conditions, the MG neuron impulse burst was prolonged, included more action potentials per burst,
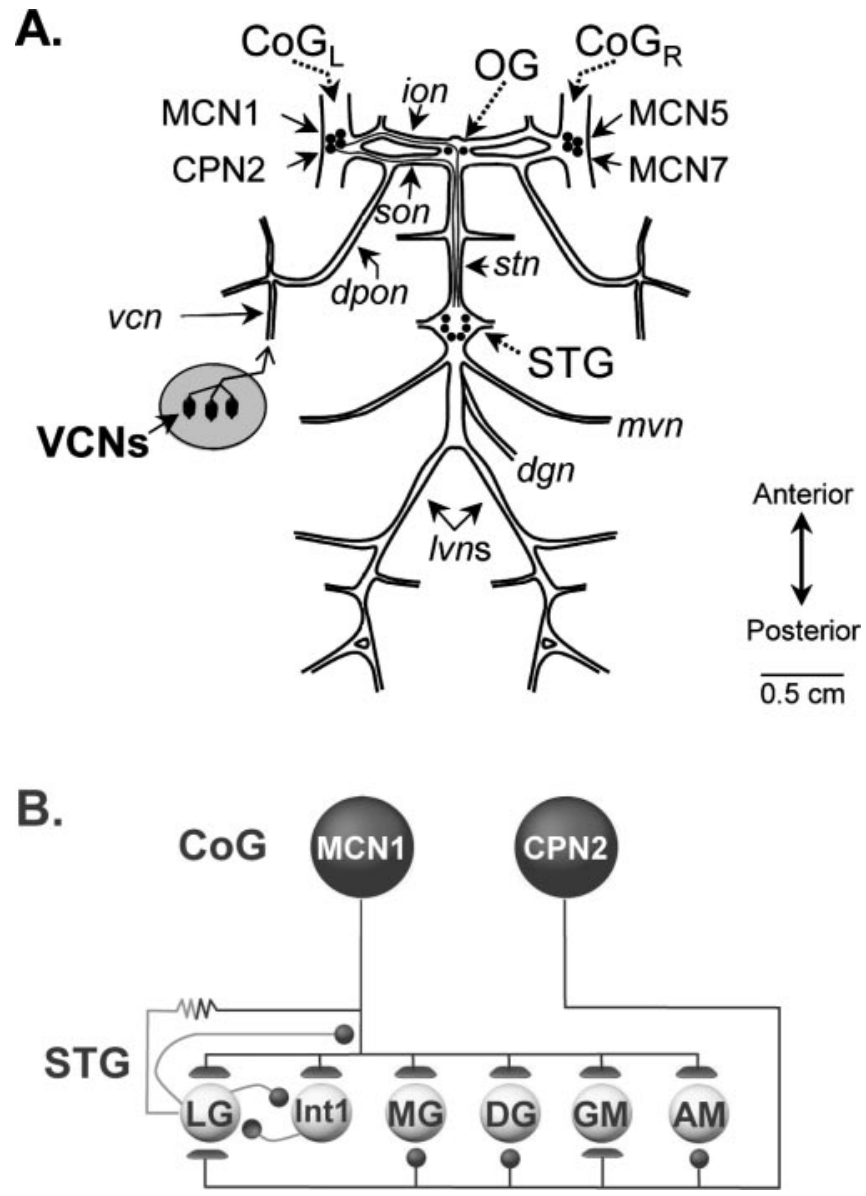

Figure 1. The isolated stomatogastric nervous system and the gastric mill circuit. $A$, The isolated stomatogastric nervous system consists of the stomatogastric ganglion (STG), oesophageal ganglion $(\mathrm{OG})$, and paired commissural ganglia (COGs) plus their connecting and peripheral nerves. All identified CoG projection neurons occur as single copies in each CoG. Each of the bilaterally symmetrical VCN mechanosensory systems projects through the vcn, dpon, and son to innervate the ipsilateral $\mathrm{C} O \mathrm{G}$. Arrows with dotted lines point to ganglia. Arrows with full lines point to nerves and identified projection neurons. B, Schematic of the identified MCN1 and CPN2 synaptic actions on the gastric mill neurons. MCN1 data were obtained from Coleman and Nusbaum (1994), Coleman et al. (1995), and Beenhakker (2004). CPN2 data were obtained from Norris et al. (1994). T-bars, synaptic excitation; filled circles, synaptic inhibition; resistor symbol, electrical coupling. Nerve labels (in italics) include the following: dgn, dorsal gastric nerve; dpon, dorsal posterior oesophageal nerve; ion, inferior oesophageal nerve; Ign, lateral gastric nerve; I $v n$, lateral ventricular nerve; $m g n$, medial gastric nerve; $m v n$, medial ventricular nerve; son, superior oesophageal nerve; stn, stomatogastric nerve; $v c n$, ventral cardiac nerve. Neuron labels include the following: AM, anterior median neuron; (PN2, commissural projection neuron 2; DG, dorsal gastric neuron; GM, gastric mill neuron; Int1, interneuron $1 ; \mathrm{LG}$, lateral gastric neuron; MCN1, 5, 7, modulatory commissural neuron 1, 5, 7; MG, medial gastric neuron; VCN, ventral cardiac neuron.

and exhibited an increased duty cycle (all parameters, $p<0.05$; $n=7$ ) (Figs. 2, 3). Each DG neuron impulse burst also exhibited an increased duty cycle, because its burst onset was phaseadvanced $(p<0.05 ; n=7)$ (Figs. 2, 3).

CPN2 also inhibits the gastropyloric inferior cardiac (IC) neuron (Norris et al., 1994), and, consistent with this action, CPN2 removal enhanced gastric mill-timed IC neuron spiking activity (Fig. 2). Specifically, the number of IC neuron spikes per burst increased after CPN2 removal (control, $0.4 \pm 1.0$ spikes/burst; CPN2 removed, $5.2 \pm 1.9$ spikes/burst; $p<0.05 ; n=6$ ) as did its intraburst firing frequency (control, $1.9 \pm 4.7 \mathrm{~Hz}$; CPN2 removed, $13.7 \pm 2.9 \mathrm{~Hz} ; p<0.05 ; n=6)$. One aspect of the gastric mill rhythm that was not altered by removing CPN2 activity was 

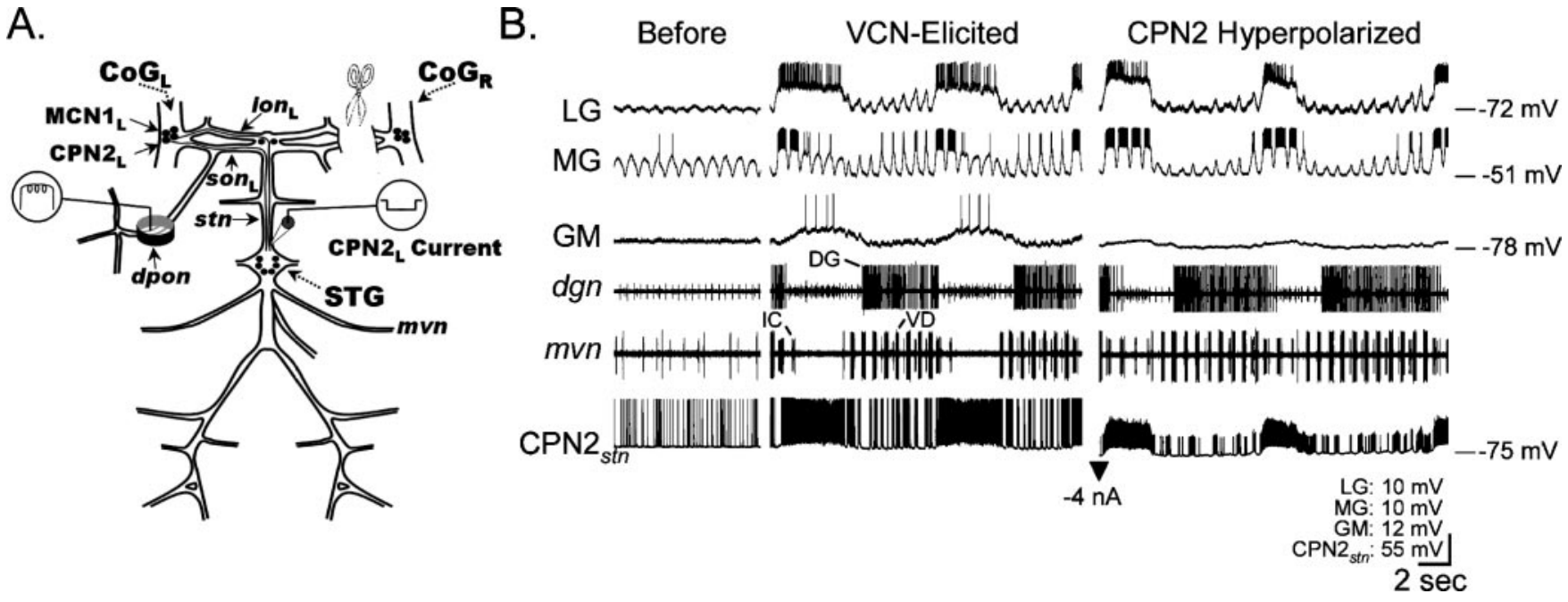

Figure 2. The VCN-elicited gastric mill rhythm is altered by selective CPN2 removal. A, Experimental manipulation was used to eliminate CPN2. An intra-axonal recording of CPN2 was made in the stn near the STG. During the VCN-elicited gastric mill rhythm the stn axon of the still-connected CPN2 was hyperpolarized sufficiently to reduce action potential amplitude, suppress its active propagation, and, consequently, remove the CPN2 influence on the STG circuits. B, The activity of gastric mill neurons before VCN stimulation (left), during a VCN-elicited gastric mill rhythm (middle), and while hyperpolarizing (PN2 during the gastric mill rhythm (right). Note the abbreviated LG neuron impulse bursts, lack of GM neuron activity, and enhanced activity of the DG, MG, and IC neurons. The amplitude of the IC neuron spikes is smaller than that of the VD neuron spikes ( $m v n$ ).

A.

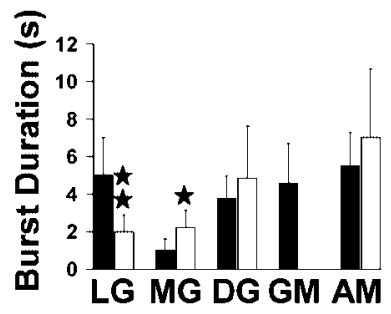

B.

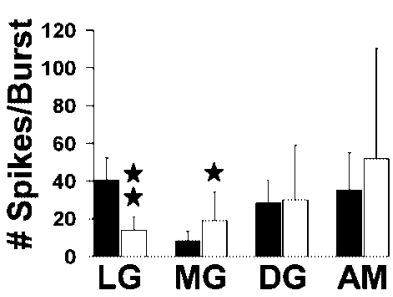

C.

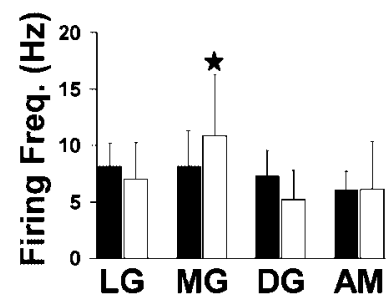

D.

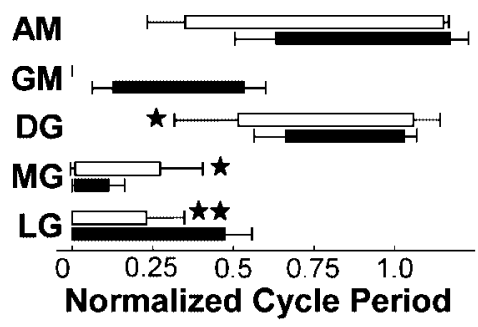

Figure 3. Quantification of VCN-elicited gastric mill rhythm parameters before and after the removal of CPN2 influence in the STG. Black bars represent data from gastric mill rhythms occurring when the MCN1 and CPN2 projection neurons influenced the STG (control data), whereas white bars represent data from rhythms in which CPN2 influence was removed selectively (experimental data). All control and experimental data were obtained from the same preparations. A, Removing (PN2 influence in the STG changed the impulse burst duration in several gastric mill neurons (LG, $n=8 ; \mathrm{MG}, n=7)$ and eliminated GM neuron bursting in seven of eight preparations. The burst durations of the $D G(n=7)$ and $A M(n=3)$ neurons were unchanged. $B$, The number of action potentials ("spikes") per impulse burst also was decreased in the LG neuron ( $p<0.001 ; n=8)$ and increased in the MG neuron ( $n=7)$ but was unchanged in the DG $(n=7)$ and $A M(n=3)$ neurons. C, The intraburst firing frequency was unchanged by CPN2 removal for most gastric mill neurons ( $L G, n=8 ; D G, n=7 ; A M, n=3)$. CPN2 removal did increase MG neuron intraburst firing frequency $(n=7)$. D, CPN2 removal phase-advanced the $L G$ neuron burst termination ( $L G, n=8)$, phase-delayed the MG neuron burst termination ( $n=7)$, and phase-advanced the onset of the DG neuron impulse burst (DG, $n=7$ ). Except as noted, ${ }^{* *} p<0.01$ and ${ }^{*} p<0.05$. All statistical analyses were performed on data derived within preparations (Wilcoxon signed rank test).

the cycle period (control, $10.5 \pm 2.7 \mathrm{sec}$; CPN2 removed, $8.7 \pm$ $2.8 \mathrm{sec} ; p>0.05 ; n=8)$. Removal of CPN2 activity during VCNelicited gastric mill rhythms thus altered many aspects of gastric mill neuron activity (Fig. 3 ).

Selective removal of MCN1 alters the VCN-elicited gastric mill rhythm

MCN1 also plays a significant role in the VCN-elicited gastric mill rhythm, as was evident from experiments in which the influence of this projection neuron on the STG was removed selectively by ion transection. MCN1 has excitatory actions on all gastric mill circuit neurons, including the gastropyloric IC and ventricular dilator (VD) neurons (Coleman and Nusbaum, 1994; Coleman et al., 1995; Bartos and Nusbaum, 1997; Blitz et al., 1999; Beenhakker, 2004) (Fig. 1B). When MCN1 was removed, the resulting VCN-elicited gastric mill rhythm included briefer impulse bursts in the LG $(p<0.01 ; n=8)$, DG $(p<0.05 ; n=6)$ and GM $(p<0.05 ; n=7)$ neurons (Figs. $4,5 A)$. MCN1 removal also resulted in fewer spikes/burst in the LG $(p<0.01 ; n=8)$ and DG $(p<0.05 ; n=6)$ neurons (Figs. $4,5 B)$. There was also a decrease in the intraburst firing frequency of the LG $(p<0.05$; $n=8)$ and DG neurons $(p<0.05 ; n=6)$ (Fig. $5 C$ ). As in control conditions, the IC neuron continued to turn off during the LG neuron burst, presumably because it continued to receive rhythmic inhibition from CPN2 $(n=4)$.

Removal of MCN1 also increased the speed of the gastric mill rhythm, as is evident from the briefer cycle period observed when MCN1 activity was eliminated (control, $11.1 \pm$ $1.9 \mathrm{sec} ; \mathrm{MCN} 1$ removal, $8.3 \pm 1.7 \mathrm{sec} ; p<0.05 ; n=8)$. Interestingly, MCN1 removal also reduced the likelihood that a gastric mill rhythm would result from VCN stimulation. A VCN-elicited gastric mill rhythm occurred in only 8 of 12 preparations $(67 \%)$ in which MCN1 was removed. In contrast, in all preparations ( 8 of 8 ) in which CPN2 activity was removed selectively, there still occurred a VCN-elicited gastric mill rhythm, albeit with an altered pattern. In those preparations in which VCN stimulation did generate a gastric mill rhythm after MCN1 removal it was clear that, like after CPN2 removal, the resulting motor pattern was distinct from the normal VCN-elicited motor pattern (Fig. 5D). 


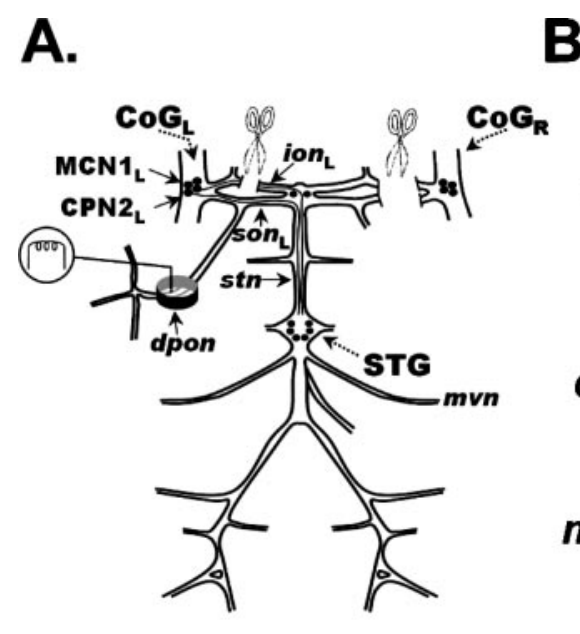

B.

\section{Before}

VCN-Elicited

m-
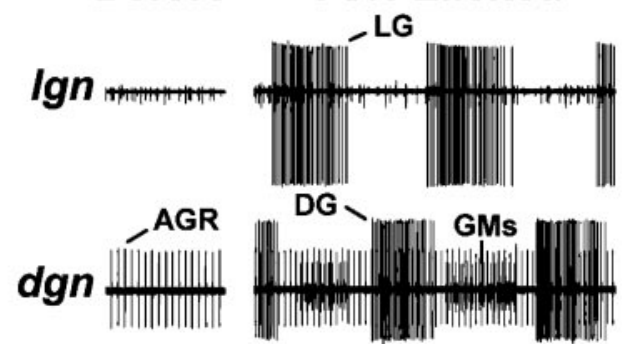

$M G^{-}$

mgn

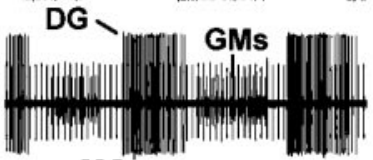

\section{ion Transected}
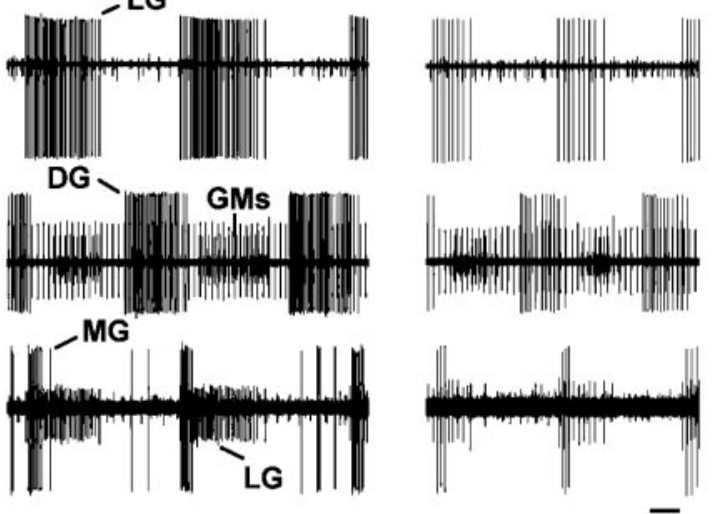

$2 \overline{\mathrm{sec}}$

Figure 4. The VCN-elicited gastric mill rhythm was altered by selective MCN1 removal. A, Experimental manipulation was used to eliminate MCN1. The influence of the single connected MCN1 was eliminated by transecting the remaining ion (see Materials and Methods). B, Gastric mill neuron activity before (left) and after (middle) VCN stimulation and after MCN1 removal (right) during the VCN-elicited gastric mill rhythm. In general, removing MCN1 activity weakened the activity levels of gastric mill neurons during the VCN-elicited gastric mill rhythm. The tonically active unit in the dgn corresponds to the activity of the anterior gastric receptor (AGR) neuron, a muscle tendon proprioceptor neuron that is spontaneously active in the isolated STNS (Combes et al., 1995).

A.

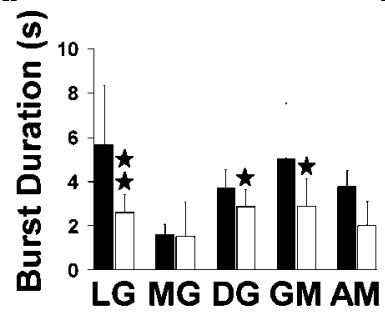

B.

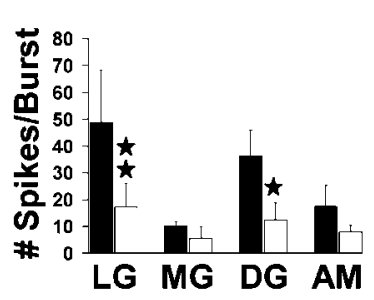

C.

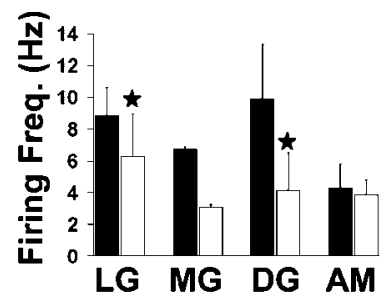

D.

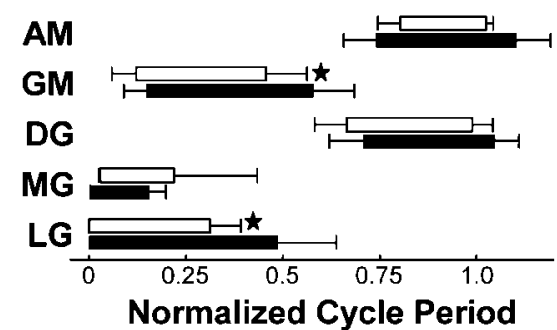

Figure 5. Quantification of gastric mill rhythm parameters before and after MCN1 removal. Black bars represent control VCN-elicited gastric mill rhythms, and the white bars represent data from gastric mill rhythms in which the MCN1 influence was removed. Both data sets were obtained from the same preparations. $A$, Removing MCN1 reduced the impulse bursts of the $L G$, DG, and GM neurons ( $\mathrm{LG}, n=8 ; \mathrm{DG}, n=6 ; \mathrm{GM}, n=7)$, but not those of the $\mathrm{MG}(n=2)$ and $\mathrm{AM}(n=4)$ neurons. $B$, The number of spikes per burst was decreased for the $\mathrm{LG}(n=8)$ and $\mathrm{DG}(n=6)$ neurons, but not for the MG $(n=2)$ and $\mathrm{AM}(n=3)$ neurons. $C$, The intraburst firing frequency was decreased for the $L G(n=8)$ and DG $(n=6)$ neurons by MCN1 removal but was not changed for the MG $(n=2)$ and $\mathrm{AM}(n=4)$ neurons. D, MCN1 removal phase-advanced the termination of the LG $(n=8)$ and GM $(n=7)$ neurons. There was no effect on the MG $(n=2)$, DG $(n=6)$, and AM $(n=$ 4) neurons. ${ }^{* *} p<0.01 ;{ }^{*} p<0.05$. All statistical analyses were performed on data derived within preparations (Wilcoxon signed rank test). The data for those neurons for which the activity was eliminated completely by MCN1 removal (MG, $n=1$ of 3 preparations; $A M, n=2$ of 6 preparations) were not included in these analyses.

MCN1 and CPN2 are necessary and probably sufficient for initiating the VCN-elicited gastric mill rhythm

Because both MCN1 and CPN2 clearly influenced the VCNelicited gastric mill rhythm, we determined whether removing the influence of both projection neurons would eliminate this rhythm. Thus we performed experiments in which the ion was transected to eliminate MCN1 influence in the STG, and the stn axon of CPN2 was hyperpolarized to eliminate the CPN2 influence therein. In these preparations stimulating the VCN sensory system failed to elicit any gastric mill rhythm-like activity $(n=6)$ (Fig. 6). In some of these experiments there were small changes in the pyloric rhythm-timed membrane potential oscillations and activity of the LG and MG neurons (Fig. 6). These pyloric-timed changes presumably resulted from the fact that the VCN system directly influences the pyloric rhythm (Beenhakker et al., 2004). It should be noted that the failure of the VCN system to elicit gastric mill rhythm-like activity after eliminating MCN1 and CPN2 influence in the STG was not likely attributable to a diminished VCN action as time progressed in an experiment because the VCN system can activate the gastric mill rhythm repeatedly in each preparation (Beenhakker et al., 2004). This result demon- strated that conjoint activation of MCN1 and CPN2 was necessary to obtain a VCN-elicited gastric mill rhythm.

We also aimed to determine whether MCN1 and CPN2 were sufficient to obtain the VCN-elicited gastric mill rhythm. We addressed this possibility by stimulating these two neurons selectively after cutting away the CoGs to remove all uncontrolled projection neuron input to the STG. To best mimic the normal MCN1 and CPN2 activity patterns, we first characterized their activity during the VCN-elicited gastric mill rhythm. Both MCN1 and CPN2 expressed stereotyped activity patterns during VCNelicited gastric mill rhythms, an example of which is shown in Figure 7. The relatively high level of activity in both MCN1 (30 \pm $4 \mathrm{~Hz} ; n=4)$ and CPN2 $(24 \pm 0.6 \mathrm{~Hz} ; n=3)$ during the gastric mill rhythm was maintained by mechanisms localized to the CoG, because these activity levels persisted when all feedback from STG neurons was removed by transecting the ions and sons ( $n=5$; data not shown). Nonetheless, feedback from STG neurons did play an important role in shaping the VCN-elicited activity pattern of both MCN1 and CPN2 as their pattern changed from rhythmic to tonic with removal of STG feedback. For example, MCN1 firing normally is regulated by the faster STG- 


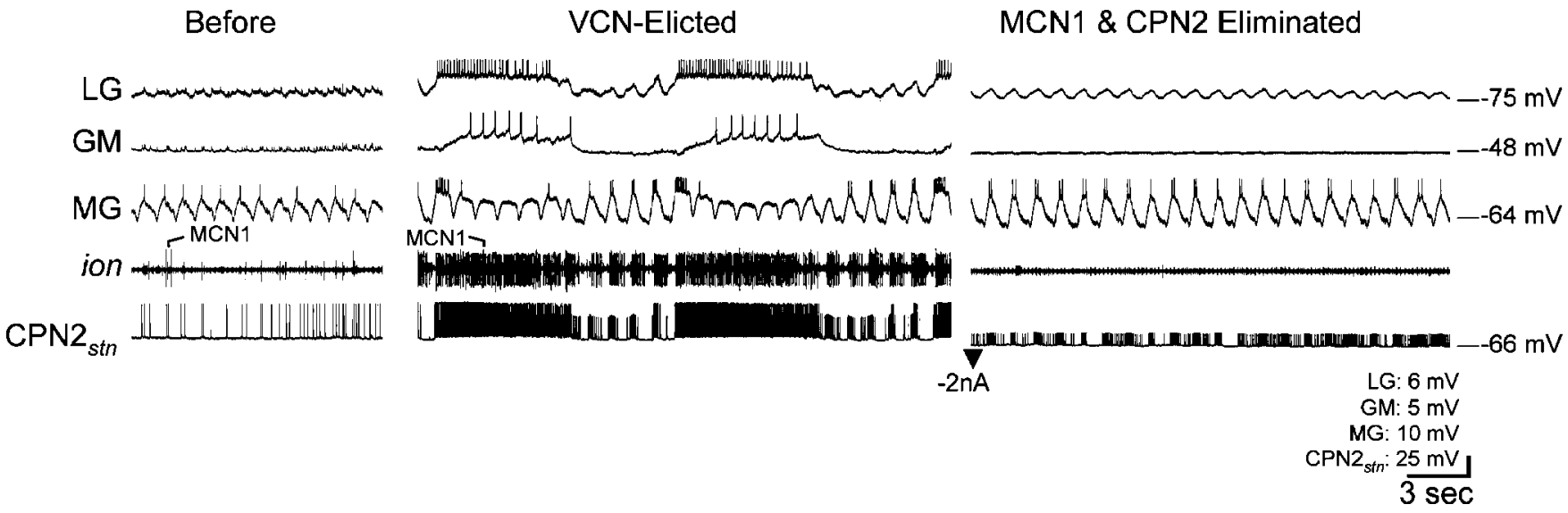

Figure 6. Simultaneous MCN1 and CPN2 removal eliminates the ability of VCN stimulation to elicit the gastric mill rhythm. Left, Before the VCN sensory pathway was stimulated in the isolated STNS, the gastric mill neurons either were inactive (LG, GM) or expressed pyloric-timed activity (MG). Note also that MCN1 and (PN2 were weakly active. Middle, Gastric mill rhythm generated by VCN stimulation. Right, Selective elimination of MCN1 (ion transection) and CPN2 (axon hyperpolarization) prevented VCN activation of the gastric mill circuit. The ion recording was on the STG side of the transection.

generated pyloric rhythm (Coleman and Nusbaum, 1994). During the VCNelicited gastric mill rhythm MCN1 activity was pyloric-timed during the LG neuron interburst instead of having the tonic activity pattern that it exhibited during each LG neuron burst (Fig. 7).

CPN2 also exhibited a stereotyped activity pattern during VCN-elicited gastric mill rhythms as a consequence of STG feedback. CPN2 is inhibited by Int1, a gastric mill pattern generator interneuron whose activity alternates with that of the LG neuron (Norris et al., 1994; Bartos et al., 1999). Because of this inhibitory influence of Int1, CPN2 was active primarily during the LG burst while its activity was weak or off during the LG interburst (Fig. 7). Thus during gastric mill rhythms elicited by the VCN sensory system, MCN1 and CPN2 expressed tonic activity during each LG neuron burst, and, during the LG neuron interburst, MCN1 exhibited pylorictimed activity whereas CPN2 was generally silent. Because the VCN-elicited gastric mill rhythm is characterized by an $\sim 10 \mathrm{sec}$ cycle period during which the LG neuron impulse burst occurs for $\sim 5 \mathrm{sec}$ (i.e., 50\% duty cycle) (Beenhakker et al., 2004), we selectively stimulated MCN1 and CPN2, using a 50\% duty cycle for their LG burst and interburst patterns.

After establishing the MCN1 and CPN2 activity patterns during VCN-elicited gastric mill rhythms, we selectively and coordinately activated these two projection neurons with these patterns to determine whether they were sufficient to elicit a VCN equivalent rhythm $(n=9)$ (Fig. 8). We assessed this possibility in preparations in which both CoGs were removed from the STNS by transecting the ions and sons (Fig. $1 \mathrm{~A}$ ). We selectively activated MCN1 by stimulating the ion (see Materials and Methods) and activated CPN2 with intra-axonal depolarizing current injection. The ion stimulation used to activate MCN1 included a $30 \mathrm{~Hz}$ tonic component (duration, $5 \mathrm{sec}$ ) that alternated with a pyloric-like rhythmic component (cycle period, 1 sec; duty cycle, $80 \%$; stimulation frequency, $30 \mathrm{~Hz}$; stimulation duration, $5 \mathrm{sec}$ ) to reflect activity expressed during the VCN-elicited gastric mill rhythm (Fig. 7).

\section{VCN-Elicited}

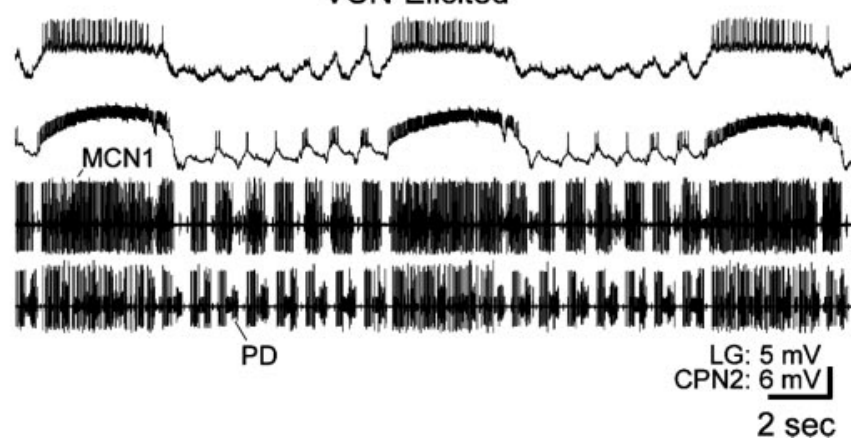

$2 \mathrm{sec}$

Figure 7. MCN1 and CPN2 exhibit gastric mill- and pyloric-timed bursts during the VCN-elicited gastric mill rhythm. MCN1 exhibited a stereotyped activity pattern that included tonic activity during each $L G$ neuron burst and pyloric-timed bursts during each $L G$ neuron interburst. The bottom recording $(d v n)$ includes the activity of the paired pyloric dilator (PD) neurons, members of VCN-elicited gastric mill rhythm was characterized predominantly by a tonic burst during each $\mathrm{L} G$ neuron burst. Most hyperpolarized membrane potential: $\mathrm{LG},-57 \mathrm{mV}$; CPN2, $-66 \mathrm{mV}$.

Intra-axonal depolarization of CPN2 also was delivered rhythmically to mimic activity during the VCN-elicited gastric mill rhythm. In general, such depolarization resembled the physiological firing pattern of CPN2. There was a difference, however, that resulted from the fact that during the VCN-elicited gastric mill rhythm each CPN2 burst of action potentials evolved over time, with the peak firing frequency occurring in the middle of the burst (Fig. 7). Thus the first third of the CPN2 burst had a relatively moderate firing frequency $(19 \pm 8 \mathrm{~Hz} ; n=3)$ compared with the middle third of the CPN2 burst $(28 \pm 9 \mathrm{~Hz} ; p<0.05 ; n=$ $3)$. The final third of the CPN2 burst had a firing frequency that was not significantly different $(24 \pm 9 \mathrm{~Hz} ; n=3 ; p>0.05)$ from that occurring during the first third of the burst. In contrast, CPN2 firing frequency in response to constant intra-axonal current injection ("one-step" current injection; Fig. $8 \mathrm{~B}$ ) was highest during the first third of the induced CPN2 burst $(26 \pm 3 ; n=4)$ and then remained relatively constant at a slightly lower firing frequency $(24 \pm 3 \mathrm{~Hz} ; p<0.05 ; n=4)$ for the remainder of the burst.

Experiments in which MCN1 and CPN2 were coactivated selectively by using the aforementioned stimulation patterns, in the absence of all other projection neuron input, elicited gastric mill rhythms that were similar to the rhythms elicited by the VCN 

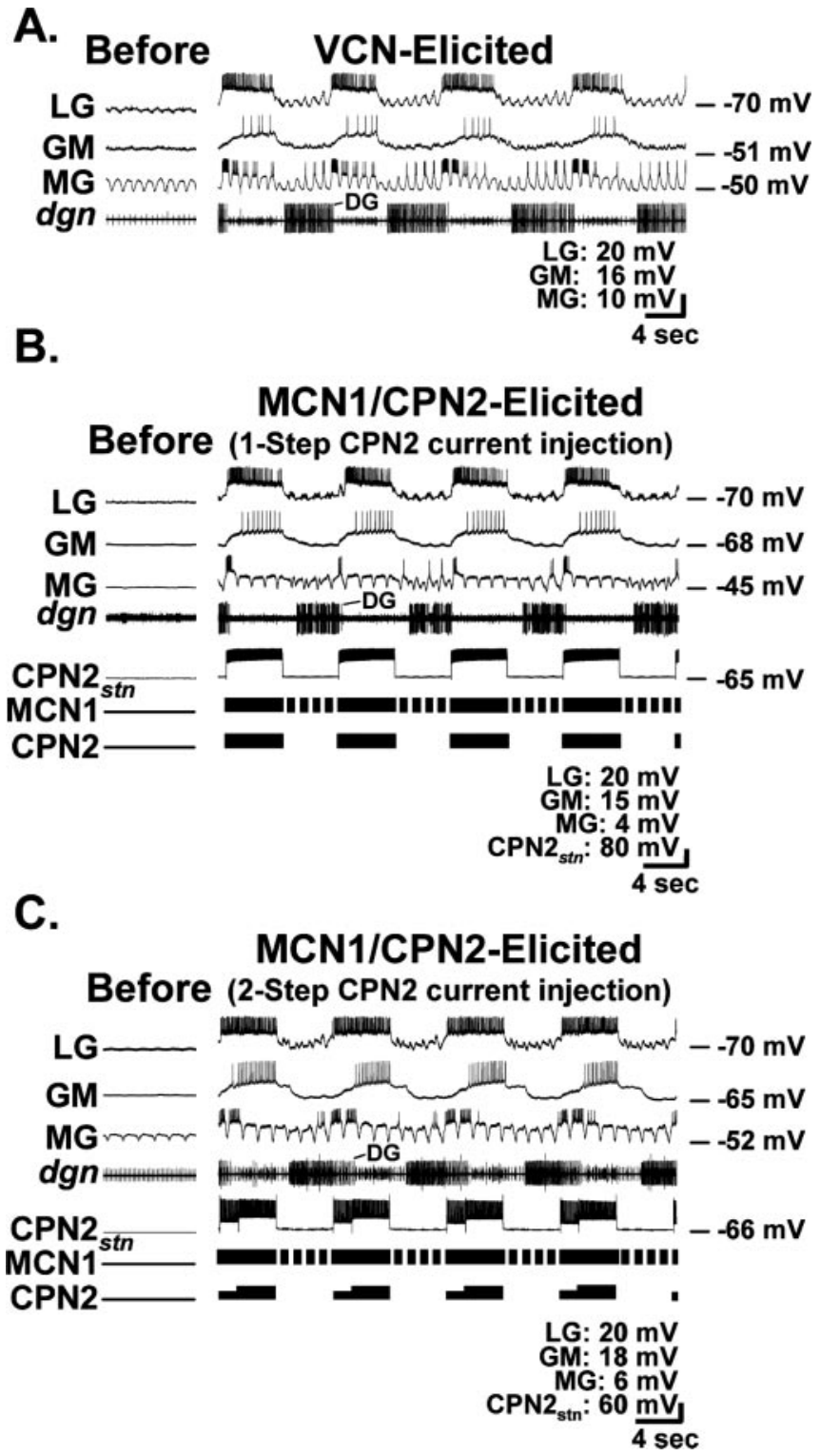

Figure 8. MCN1 and CPN2 activity is sufficient to elicit a VCN-like gastric mill rhythm. A, The gastric mill rhythm was not spontaneously active, but VCN stimulation in such preparations elicited the gastric mill rhythm. $B$, In preparations in which both CoGs were removed, selective activation of MCN1 (ion stimulation, bars) and CPN2 (intra-axonal depolarizing current injection, bars), using their gastric mill rhythm patterns, elicited a VCN-like gastric mill rhythm (see Results for details). C, An example of an experiment similar to that described in $B$ but with a two-step depolarizing current injection delivered to the stn axon of CPN2 to mimic better the (PN2 activity observed during the VCN-elicited gastric mill rhythm (see Results for details).

sensory system (Figs. 8, 9). The VCN-elicited gastric mill rhythms that we used for the control data set $(n=13)$ were those that we obtained for the previous experiments in this work. Because they were similar in all regards $(p>0.05)$, these data were pooled and used as the control group to which the MCN1/CPN2-elicited gastric mill rhythms were compared. Quantification of various aspects of gastric mill neuron activity showed that the resulting one-step MCN1/CPN2-elicited gastric mill rhythm was indistinguishable from the VCN-elicited rhythm on most parameters (Figs. $8 B, 9$ ). For example, these two rhythms had statistically indistinguishable cycle periods (control, $10.8 \pm 2.3 \mathrm{sec}, n=13$; one-step MCN1/CPN2, $10.0 \pm 0.9 \mathrm{sec}, n=9 ; p>0.05)$. Furthermore, all analyzed gastric mill neurons had comparable burst durations and number of spikes per burst during both rhythms $(p>0.05)$. Two of the four analyzed neurons (LG, MG) had unchanged intraburst firing frequencies as well. However, there was an increased intraburst firing frequency in the DG neuron ( $p<0.05$; MCN1/CPN2-elicited, $n=9$; VCN-elicited, $n=13$ ) and a decreased anterior median (AM) neuron firing frequency $(p<0.05$; MCN1/CPN2-elicited, $n=5$; VCN-elicited, $n=9)$ during the MCN1/CPN2-elicited gastric mill rhythm. In most instances the phase relationships of the gastric mill neurons were the same. However, the termination of the DG neuron impulse burst was phase-advanced during the MCN1/CPN2-elicited gastric mill rhythm relative to the VCN-elicited gastric mill rhythm ( $p<0.05$; MCN1/CPN2-elicited, $n=9$; VCN-elicited, $n=13$ ), as was the onset of the burst generated by the GM neuron $(p<$ 0.05; MCN1/CPN2-elicited, $n=9$; VCN-elicited, $n=13$ ).

In subsequent experiments we tested the hypothesis that the differences between the VCN-elicited and the one-step MCN1/ CPN2-elicited gastric mill rhythms resulted from our inadequate version of the physiological firing pattern of CPN2 during the MCN1/CPN2-elicited gastric mill rhythm. During the VCNelicited gastric mill rhythm the CPN2 burst is characterized by a gradual increase in firing frequency (Fig. 7), such that its peak firing frequency occurs $\sim 1.5 \mathrm{sec}$ after burst onset. In contrast, CPN2 firing frequency in response to constant-amplitude current injection occurs during the initial portion of the burst. Thus to approximate better the CPN2 burst observed during VCNelicited gastric mill rhythms, we generated MCN1/CPN2-elicited gastric mill rhythms with a two-step depolarizing current injection into the stn axon of CPN2 (Fig. 8C). The initial $(0-1.5 \mathrm{sec})$ depolarizing current injection was smaller in amplitude (1-2 nA) than the remaining $(1.5-5 \mathrm{sec})$ depolarizing current injection (3-5 nA), yielding a peak CPN2 firing frequency that occurred $\sim 1.5 \mathrm{sec}$ after burst onset (CPN2 firing frequency during first current injection step, $18.4 \pm 2.4 \mathrm{~Hz}$; CPN2 firing frequency during second current injection step, $23.3 \pm 4.5 \mathrm{~Hz} ; n=5$ ). Altering the CPN2 burst in this manner produced a MCN1/ CPN2-elicited gastric mill rhythm that better resembled the VCN-elicited gastric mill rhythm (Figs. $8 C, 9$ ). The only remaining differences occurred in the number of DG neuron spikes per burst and its intraburst firing frequency (both parameters, $p<$ 0.05; MCN1/CPN2-elicited, $n=4$; VCN-elicited, $n=13)$. The cycle period of the two-step MCN1/CPN2-elicited gastric mill rhythm $(9.8 \pm 0.04 \mathrm{sec})$ was not different $(p>0.05)$ from that of either the one-step or VCN-elicited conditions (two-step MCN1/ CPN2-elicited, $n=5$ ).

\section{Discussion}

We have identified the projection neurons by which a specific sensory system activates a rhythmically active motor circuit. Previous work showed that the VCN mechanosensory system influences at least four CoG projection neurons (Beenhakker et al., 2004). All four of these neurons have synaptic actions on gastric mill neurons (Coleman and Nusbaum, 1994; Norris et al., 1994, 1996; Blitz et al., 1999). In the current study we demonstrate that, despite these relatively widespread VCN actions, the conjoint activity of MCN1 and CPN2 is necessary and likely sufficient to mediate the VCN-elicited gastric mill rhythm. Necessity was demonstrated by the failure of VCN stimulation to activate the gastric mill rhythm after selective removal of MCN1 and CPN2. The likely sufficiency of MCN1 and CPN2 was demonstrated by the VCN-like gastric mill rhythm elicited by the selective coactivation of these two projection neurons. One remaining caveat is that the current injections used to manipulate CPN2 activity also 
A.

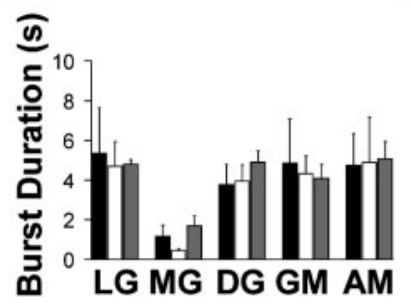

B.

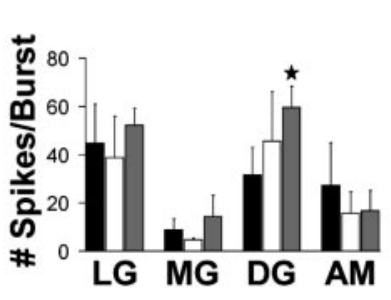

C.

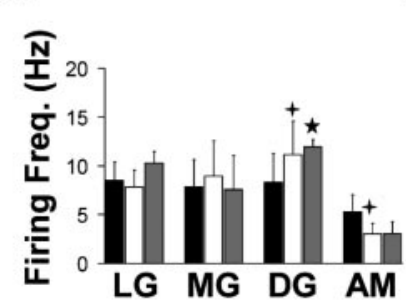

D.

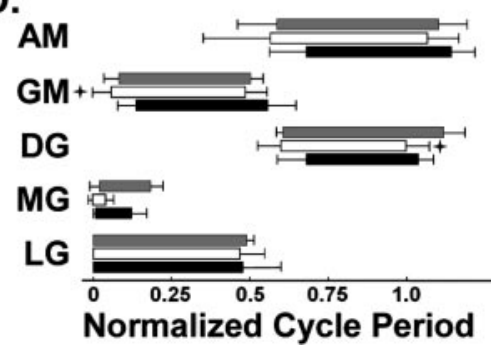

Figure 9. Quantification of the VCN-elicited gastric mill rhythm (black bars, control rhythms) and comparison with the rhythm elicited by MCN1/CPN2 stimulation (white bars, one-step CPN2 current injection; gray bars, two-step (PN2 current injection). $A$, The burst durations of the gastric mill neurons were not different among the VCN-elicited (LG, $n=16$; MG, $\mathrm{AM}, n=9 ; \mathrm{DG}, \mathrm{GM}, n=$ 13), the one-step MCN1/CPN2-elicited (LG, DG, GM, $n=9 ; \mathrm{MG}, n=3 ; \mathrm{AM}, n=5$ ), and the two-step MCN1/CPN2-elicited (LG, MG, $n=5 ; \mathrm{DG}, \mathrm{GM}, \mathrm{AM}, n=4)$ gastric mill rhythms. $B$, The numbers of spikes per burst generated by the gastric mill neurons were not different among these three rhythms, except for the DG neuron during the tw0-step MCN1/CPN2-elicited gastric mill relative to the control rhythm. All $n$ values are the same as in $A$. C, During the control rhythm the intraburst firing frequency of the DG neuron was lower, whereas the AM neuron firing frequency was higher than during the one-step MCN1/CPN2-elicited rhythm. During the two-step MCN1/CPN2-elicited gastric mill rhythm only the DG neuron intraburst firing frequency was distinct from the control rhythm. All $n$ values are the same as in A.D, During the one-step MCN1/CPN2-elicited gastric mill rhythm the termination of the DG neuron impulse burst and the onset of the GM burst were phase-advanced relative to the control rhythm. All of the phase relationships among the gastric mill neurons during the two-step MCN1/CPN2-elicited gastric mill rhythm were the same as during the control rhythm. All $n$ values are the same as in A. Data were compared across preparations; therefore, data analysis was performed by using ANOVAs (Kruskal-Wallis). ${ }^{*} p<0.05$; a plus symbol signifies differences between the one-step MCN1/CPN2-elicited and the VCN-elicited gastric mill rhythm; filled star signifies differences between the two-step MCN1/CPN2-elicited and the VCN-elicited gastric mill rhythm.

may alter the activity of other projection neurons if they are coupled electrically to CPN2 in the STG. At this point, however, there is no evidence supporting this possibility.

The MCN1/CPN2- and VCN-elicited gastric mill rhythms were not identical. The few differences between these two rhythms may have resulted from our inability to mimic exactly the physiological firing pattern of CPN2. The mismatch in CPN2 activity likely resulted from the presence of regenerative properties in the CoG where CPN2 bursts normally originate (Norris et al., 1994) and the absence of these properties in its stn axon. The differences between the VCN- and MCN1/CPN2-elicited gastric mill rhythms are consistent with the occurrence of an earlier-thannormal onset of high-frequency firing in CPN2 and its resulting strengthened synaptic actions during intra-axonal current injection (Norris et al., 1994). For example, the phase-advanced GM burst probably results from the strengthened excitatory input from CPN2 early in the cycle. This strengthened CPN2 activity also would inhibit the DG and AM neurons for a longer duration and could alter their post-inhibitory rebound burst properties.

When the current injection protocol used to activate CPN2 was altered to approximate better the physiological CPN2 firing pattern, the resulting MCN1/CPN2-elicited gastric mill rhythm was more similar to the VCN-elicited gastric mill rhythm, differing only in the number of spikes and intraburst firing frequency of the DG neuron. These remaining differences may have resulted from the different basal modulatory state of the STG that likely exists between the two experimental approaches. For example, the VCN-elicited gastric mill rhythm occurred in preparations in which the CoGs were connected to the STG, providing the STG with some basal level of modulatory input resulting from spontaneous activity of some CoG projection neurons (Nagy and Cardi, 1994; Nagy et al., 1994). The MCN1/CPN2-elicited gastric mill rhythm, on the other hand, occurred in preparations with the CoGs removed. However, the possibility also remains that a third, unidentified CoG projection neuron contributed to the altered DG neuron activity observed during the VCN-elicited gastric mill rhythm. If there is such a neuron, it is unlikely to be MCN5 or MCN7 (Beenhakker et al., 2004; this work).

With the identification of most or all of the projection neurons responsible for the VCN-elicited gastric mill rhythm, it becomes possible to determine the contribution of each participat- ing projection neuron to the activation of the gastric mill circuit. Experiments in which either MCN1 or CPN2 was removed selectively suggest that the relative contributions of these two projection neurons to the VCN-elicited gastric mill rhythm reflect their previously characterized actions on gastric mill neurons. The contribution of MCN1 includes a modulatory excitation of some neurons (e.g., LG, DG) and ionotropic excitation of others (e.g., Int1) (Coleman and Nusbaum, 1994; Coleman et al., 1995; Wood et al., 2000). These actions contribute to the ability of MCN1 stimulation to drive the gastric mill rhythm (Coleman et al., 1995; Bartos et al., 1999). The CPN2 contribution includes fast excitation of the LG and GM neurons and inhibition of the DG, AM, IC, and MG neurons. These actions are also sufficient to elicit a coordinated gastric mill rhythm (Norris et al., 1994; this work). MCN1 probably provides the main drive to the gastric mill circuit whereas CPN2 serves to sculpt gastric mill neuron activity, because MCN1-only preparations, but not CPN2-only preparations, faithfully generated gastric mill rhythms. The likelihood that MCN1 provides the primary drive for the gastric mill rhythm is supported by previous studies (Norris et al., 1994; Coleman et al., 1995; Bartos et al., 1999). It was clear, however, that only the collective actions of both projection neurons were sufficient to enable a gastric mill rhythm similar to that elicited by the VCN pathway.

Many sensory systems directly regulate the timing and activity levels of CPG neurons (Stein et al., 1997). It also has become clear that sensory systems can influence the upstream neurons, such as reticulospinal neurons, that project to and regulate CPG activity (Viana di Prisco et al., 1997, 2000; Li et al., 2003). Despite the relatively rich cellular level understanding underlying the activation of some reticulospinal neuron subtypes by a sensory system, current experimental approaches limit our understanding of how that sensory information is represented across the population of reticulospinal neurons.

The challenge in identifying the full complement of projection neurons mediating the actions of a sensory system is exacerbated by the possibility of false positive results. False positives can result from the fact that selective stimulation of single projection neurons can elicit complete rhythmic motor patterns (Wiersma and Ikeda, 1964; Eaton et al., 1977; Weeks and Kristan, 1978; Nagy and Dickinson, 1983; Rosen et al., 1991; Frost and Katz, 1996; 
Kemenes et al., 2001). In C. borealis alone there are at least five projection neurons that elicit different pyloric rhythms when individually stimulated (Norris et al., 1996; Blitz et al., 1999; Christie et al., 2004). With this in mind, sensory regulation of a neuronal circuit could result from its activation of a single projection neuron, a subset of relevant projection neurons, or all relevant projection neurons. For example, the reticulospinal Mauthner cell in fish was thought to act as a "command neuron" that, when excited by sensory inputs, activated an entire escape motor program. However, subsequent work revealed that other reticulospinal neurons contribute to such behaviors (Liu and Fetcho, 1999; Gahtan et al., 2002).

Previous work in the lobster STNS has provided a glimpse into this issue by demonstrating that a sensory system can influence a motor circuit by having distinct actions on different projection neurons. Specifically, the proprioceptive anterior gastric receptor (AGR) has different activity-dependent actions on two identified projection neurons that enable it to alter spontaneously active gastric mill rhythms in different ways, depending on its firing rate (Combes et al., 1999a,b). Although it remains unknown whether additional projection neurons are involved, this work reinforces the hypothesis that sensory-evoked activity patterns generated by STG motor circuits are at least partly a function of activity generated by subsets of upstream projection neurons.

With respect to how a sensory signal is transduced into a particular neuronal output, evidence from several systems supports the notion that the "code" is represented by the activity levels and patterns across the population of neurons responsible for eliciting that circuit activity (Berkowitz and Stein, 1994a,b; Laurent, 1996, 1997; Kristan and Shaw, 1997; Lewis, 1999; Gahtan et al., 2002). This coding scheme has received the most attention in studies involving a directed response (Georgopoulos et al., 1986; Sparks, 1988; Georgopoulos, 1995; Lewis and Kristan, 1998a,b; Lewis, 1999), but it can be extended to include categorically distinct behaviors (Kristan and Shaw, 1997). A recent hypothesis proposes that different motor behaviors result from different combinations of activity patterns in overlapping but distinct combinations of multifunctional neuronal inputs to motor circuits (Kristan and Shaw, 1997; Shaw and Kristan, 1997). With respect to sensory-evoked activation of the descending reticulospinal neuron system that activates the spinal locomotor network or the VCN-evoked activation of projection neurons that activate the gastric mill circuit, the resulting network and behavioral output also result from activity that is represented across a population of descending inputs.

In conclusion, we have shown that the neuronal circuit output in response to the activation of a particular sensory system results from the specific actions of a distinct subset of available projection neurons. It is already evident that there are multiple versions of the gastric mill rhythm (Coleman and Nusbaum, 1994; Norris et al., 1994; Beenhakker et al., 2004). However, to demonstrate that the gastric mill circuit is truly under the control of a code that is represented by overlapping but distinct populations of upstream projection neurons, the actions of several different sensory systems on those projection neurons, as well as the consequences of such actions for the gastric mill circuit, must be examined next.

\section{References}

Bartos M, Nusbaum MP (1997) Intercircuit control of motor pattern modulation by presynaptic inhibition. J Neurosci 17:2247-2256.

Bartos M, Manor Y, Nadim F, Marder E, Nusbaum MP (1999) Coordination of fast and slow rhythmic neuronal circuits. J Neurosci 19:6650-6660.
Beenhakker MP (2004) Sensory regulation of rhythmically active neuronal networks. PhD thesis, University of Pennsylvania.

Beenhakker MP, Hertzberg S, Nusbaum MP (2000) Neural network modulation by mechanosensory activation. Soc Neurosci Abstr 26:163.2.

Beenhakker MP, Blitz DM, Nusbaum MP (2004) Long-lasting activation of rhythmic neuronal activity by a novel mechanosensory system in the crustacean stomatogastric nervous system. J Neurophysiol 91:78-91.

Berkowitz A, Stein PS (1994a) Activity of descending propriospinal axons in the turtle hindlimb enlargement during two forms of fictive scratching: broad tuning to regions of the body surface. J Neurosci 14:5089-5104.

Berkowitz A, Stein PS (1994b) Activity of descending propriospinal axons in the turtle hindlimb enlargement during two forms of fictive scratching: phase analyses. J Neurosci 14:5105-5119.

Blitz DM, Nusbaum MP (1997) Motor pattern selection via inhibition of parallel pathways. J Neurosci 17:4965-4975.

Blitz DM, Christie AE, Coleman MJ, Norris BJ, Marder E, Nusbaum MP (1999) Different proctolin neurons elicit distinct motor patterns from a multifunctional neuronal network. J Neurosci 19:5449-5463.

Christie AE, Stein W, Quinlan JE, Beenhakker MP, Marder E, Nusbaum MP (2004) Actions of a histaminergic/peptidergic projection neuron on rhythmic motor patterns in the stomatogastric nervous system of the crab Cancer borealis. J Comp Neurol 469:153-169.

Coleman MJ, Nusbaum MP (1994) Functional consequences of compartmentalization of synaptic input. J Neurosci 14:6544-6552.

Coleman MJ, Nusbaum MP, Cournil I, Claiborne BJ (1992) Distribution of modulatory inputs to the stomatogastric ganglion of the crab, Cancer borealis. J Comp Neurol 325:581-594.

Coleman MJ, Meyrand P, Nusbaum MP (1995) A switch between two modes of synaptic transmission mediated by presynaptic inhibition. Nature 378:502-505.

Combes D, Simmers AJ, Moulins M (1995) Structural and functional characterization of a muscle tendon proprioceptor in lobster. J Comp Neurol 363:221-234.

Combes D, Meyrand P, Simmers J (1999a) Motor pattern specification by dual descending pathways to a lobster rhythm-generating network. J Neurosci 19:3610-3619.

Combes D, Meyrand P, Simmers J (1999b) Dynamic restructuring of a rhythmic motor program by a single mechanoreceptor neuron in lobster. J Neurosci 19:3620-3628.

Eaton RC, Bombardieri RA, Meyer DL (1977) The Mauthner-initiated startle response in teleost fish. J Exp Biol 66:65-81.

Frost WN, Katz PS (1996) Single neuron control over a complex motor program. Proc Natl Acad Sci USA 93:422-426.

Gahtan E, Sankrithi N, Campos JB, O’Malley DM (2002) Evidence for a widespread brain stem escape network in larval zebrafish. J Neurophysiol $87: 608-614$

Georgopoulos AP (1995) Current issues in directional motor control. Trends Neurosci 18:506-510.

Georgopoulos AP, Schwartz AB, Kettner RE (1986) Neuronal population coding of movement direction. Science 233:1416-1419.

Gold JI, Shadlen MN (2001) Neural computations that underlie decisions about sensory stimuli. Trends Cogn Sci 5:10-16.

Harris-Warrick RM, Marder E, Selverston AI, Moulins M, eds (1992) Dynamic biological networks. The stomatogastric nervous system. Cambridge, MA: MIT.

Hooper SL, Moulins M, Nonnotte L (1990) Sensory input induces longlasting changes in the output of the lobster pyloric network. J Neurophysiol 64:1555-1573.

Katz PS, Eigg MH, Harris-Warrick RM (1989) Serotonergic/cholinergic muscle receptor cells in the crab stomatogastric nervous system. I. Identification and characterization of the gastropyloric receptor cells. J Neurophysiol 62:558-570.

Kemenes G, Staras K, Benjamin PR (2001) Multiple types of control by identified interneurons in a sensory-activated rhythmic motor pattern. J Neurosci 21:2903-2911.

Kristan WB, Shaw BK (1997) Population coding and behavioral choice. Curr Opin Neurobiol 7:826-831.

Laurent G (1996) Dynamical representation of odors by oscillating and evolving neural assemblies. Trends Neurosci 19:489-496.

Laurent G (1997) Olfactory processing: maps, time, and codes. Curr Opin Neurobiol 7:547-553. 
Lewis JE (1999) Sensory processing and the network mechanisms for reading neuronal population codes. J Comp Physiol [A] 185:373-378.

Lewis JE, Kristan WB (1998a) A neuronal network for computing population vectors in the leech. Nature 391:76-79.

Lewis JE, Kristan WB (1998b) Representation of touch location by a population of leech sensory neurons. J Neurophysiol 80:2584-2592.

Li WC, Perrins R, Walford A, Roberts A (2003) The neuronal targets for GABAergic reticulospinal inhibition that stops swimming in hatchling frog tadpoles. J Comp Physiol [A] 189:29-37.

Liu KS, Fetcho JR (1999) Laser ablations reveal functional relationships of segmental hindbrain neurons in zebrafish. Neuron 23:325-335.

Meyrand P, Simmers J, Moulins M (1994) Dynamic construction of a neural network from multiple pattern generators in the lobster stomatogastric nervous system. J Neurosci 14:630-644.

Nagy F, Cardi P (1994) A rhythmic modulatory gating system in the stomatogastric nervous system of Homarus gammarus. II. Modulatory control of the pyloric CPG. J Neurophysiol 71:2490-2502.

Nagy F, Dickinson PS (1983) Control of a central pattern generator by an identified modulatory interneurone in crustacea. I. Modulation of the pyloric motor output. J Exp Biol 105:33-58.

Nagy F, Cardi P, Cournil I (1994) A rhythmic modulatory gating system in the stomatogastric nervous system of Homarus gammarus. I. Pyloric-related neurons in the commissural ganglia. J Neurophysiol 71:2477-2489.

Norris BJ, Coleman MJ, Nusbaum MP (1994) Recruitment of a projection neuron determines gastric mill motor pattern selection in the stomatogastric nervous system of the crab, Cancer borealis. J Neurophysiol 72:1451-1463.

Norris BJ, Coleman MJ, Nusbaum MP (1996) Pyloric motor pattern modification by a newly identified projection neuron in the crab stomatogastric nervous system. J Neurophysiol 75:97-108.

Nusbaum MP, Beenhakker MP (2002) A small-systems approach to motor pattern generation. Nature 417:343-350.

Nusbaum MP, Blitz DM, Swensen AM, Wood D, Marder E (2001) The roles of co-transmission in neural network modulation. Trends Neurosci 24:146-154.

Pouget A, Dayan P, Zemel R (2000) Information processing with population codes. Nat Rev Neurosci 1:125-132.
Rosen SC, Teyke T, Miller MW, Weiss KR, Kupfermann I (1991) Identification and characterization of cerebral-to-buccal interneurons implicated in the control of motor programs associated with feeding in Aplysia. J Neurosci 11:3630-3655.

Shaw BK, Kristan WB (1997) The neuronal basis of the behavioral choice between swimming and shortening in the leech: control is not selectively exercised at higher circuit levels. J Neurosci 17:786-795.

Simmers J, Moulins M (1988a) A disynaptic sensorimotor pathway in the lobster stomatogastric system. J Neurophysiol 59:740-756.

Simmers J, Moulins M (1988b) Nonlinear interneuronal properties underlie integrative flexibility in a lobster disynaptic sensorimotor pathway. J Neurophysiol 59:757-777.

Sparks DL (1988) Population coding of saccadic eye movements in the superior colliculus. Nature 332:357-360.

Stein PSG, Grillner S, Selverston AI, Stuart DG, editors (1997) Neurons, networks, and motor behavior. Cambridge, MA: MIT.

Viana di Prisco G, Pearlstein E, Robitaille R, Dubuc R (1997) Role of sensory-evoked NMDA plateau potentials in the initiation of locomotion. Science 278:1122-1125.

Viana di Prisco G, Pearlstein E, Le Ray D, Robitaille R, Dubuc R (2000) A cellular mechanism for the transformation of a sensory input into a motor command. J Neurosci 20:8169-8176.

Weeks JC, Kristan WB (1978) Initiation, maintenance, and modulation of swimming in the medicinal leech by the activity of a single neurone. J Exp Biol 77:71-88.

Weimann JM, Meyrand P, Marder E (1991) Neurons that form multiple pattern generators: identification and multiple activity patterns of gastric/ pyloric neurons in the crab stomatogastric system. J Neurophysiol 65:111-122.

Wiersma CA, Ikeda K (1964) Interneurons commanding swimmeret movements in the crayfish, Procambarus clarkii (Girard). Comp Biochem Physiol 12:509-525.

Wood DE, Stein W, Nusbaum MP (2000) Projection neurons with shared cotransmitters elicit different motor patterns from the same neuronal circuit. J Neurosci 20:8943-8953. 